\title{
State Observers for Systems Subject to Bounded Disturbances Using Quadratic Boundedness
}

\author{
Angelo Alessandri, Francesca Boem
}

\begin{abstract}
Quadratic boundedness is adopted to construct state observers for linear, piecewise linear, and Lipschitz nonlinear systems subject to bounded disturbances. Upper bounds on the estimation error are derived by exploiting quadratic boundedness and a design method based on linear matrix inequalities is proposed to minimize such bounds. Simulation results are provided to show the effectiveness of the proposed approach.
\end{abstract}

\section{INTRODUCTION}

The most popular way to perform estimation is by far the Kalman filter [1]. Indeed, it is pretty well-known too that its performances can degrade due to the wrong tuning of the noise covariance matrices of the system model since also such matrices may be subject to uncertainties. To overcome such difficulties, other approaches based on $\mathrm{H}_{2} / \mathrm{H}_{\infty}$ estimation have been developed in the seventies by assuming noises with bounded energy [2]. In this paper, we propose a novel design method to construct Luenberger state observers [3] for various types of continuous-time dynamic system, including piecewise linear (PWL) and Lipschitz nonlinear systems. We assume to have at disposal bounds on the system and measurement disturbances, which are explicitly exploited for the purpose of observer design by relying on quadratic boundedness (QB) [4].

The potential of dealing with estimation with bounded noises is pretty well-known [5]. The information available on the boundedness of the disturbances is not usually taken into account, as pointed out in [6]. This has motivated the development of novel optimization approaches such as those presented in [7]. However, the effectiveness of the resulting estimation methods is still a topic under investigation. In this context, we address the use of QB, which allows to deal with positively invariant sets and enables to provide upper bounds on the trajectories of the state of a system subject to bounded disturbances [4]. To the authors' knowledge, QB has been applied only to discrete-time systems for the purpose of output feedback control [8] and estimation [9], [10]. The proposed approach based on QB allows to explicitly take into account the bounds on the noises that could easily be available by the plant operators for monitoring purposes. This information is more helpful and easily used, compared to statistics or energy of the noises. The knowledge of the bounds on system and measurement disturbances is embedded in the proposed design procedure without the need of a "trial-and-error" tuning.

A. Alessandri is with the Department of Mechanical Engineering, University of Genoa, Via Opera Pia 15, 16145 Genoa, Italy e-mail: alessandri@dime.unige.it.

F. Boem is with the Department of Electronic and Electrical Engineering, University College London, UK e-mail: f.boem@ucl.ac.uk.
The main advantage of $\mathrm{QB}$ consists in deriving upper bounds on the estimation error that can be treated by using linear matrix inequalities (LMIs). This makes possible a convenient design that explicitly takes into account the boundedness of the disturbances and, thanks to convexity, it can be solved by means of well-established semi-definite programming (SDP) tools [11]. Based on the preliminary results presented in [12], in this paper we address state estimation for PWL [13], [14] and Lipschitz nonlinear systems [15]-[18]. Looking at such a literature, few methods have been investigated to estimate the state of dynamic systems by explicitly accounting for the knowledge of bounds on the noises. Among such methods, one can resort to moving horizon estimation by suitably constraining the estimated disturbances [19], [20]. Of course, this demands an increased computational burden, thus requiring the use of fast techniques [21]. As compared to moving horizon estimation, the proposed method does not require on-line optimization since the boundedness of the noises is considered in the off-line design.

The paper is organized as follows. In Section II we will present the proposed approach to observer design for LTI (linear time-invariant) and PWL systems based on QB, while the extension to systems having in addition Lipschitz nonlinearities is detailed in Section III. Simulations results are reported in Section IV. Finally, the conclusions are drawn in Section $\mathrm{V}$.

Let $(x, y):=\left[x^{\top}, y^{\top}\right]^{\top}$, where $x$ and $y$ are column vectors and the symbol ${ }^{\top}$ means transposition. The minimum and maximum eigenvalues of a real, symmetric matrix $P \in \mathbb{R}^{n \times n}$ are denoted by $\lambda_{\min }(P)$ and $\lambda_{\max }(P)$, respectively. Moreover, $P>0(P<0)$ means that it is also positive (negative) definite; $P \geq 0$ ( $P \leq 0)$ denotes that it is positive (negative) semidefinite. Given a generic matrix $M \in \mathbb{R}^{n \times m}$, $|M|:=\left(\lambda_{\max }\left(M^{\top} M\right)\right)^{1 / 2}=\left(\lambda_{\max }\left(M M^{\top}\right)\right)^{1 / 2}$ and hence, for a vector $v \in \mathbb{R}^{n},|v|:=\left(v^{\top} v\right)^{1 / 2}$ is its Euclidean norm. Moreover, $\operatorname{diag}(v) \in \mathbb{R}^{n \times n}$ is the diagonal matrix with $v_{1}, \ldots, v_{n}$ on the diagonal. The symbol $\star$ in a block matrix is used to denote completion trough symmetry.

\section{QB FOR ESTIMATION OF LTI AND PWL SYSTEMS}

In this section, after showing how to apply QB to design observers for LTI systems, we will extend the approach to PWL systems.

\section{A. LTI systems}

Let us consider the dynamic system

$$
\begin{aligned}
& \dot{x}=A x+B u+D w \\
& y=C x+E w
\end{aligned}
$$


where $t \geq 0, x(t) \in \mathbb{R}^{n}$ is the state, $u(t) \in \mathbb{R}^{p}$ is the control input, $y(t) \in \mathbb{R}^{m}$ is the output; $w(t) \in \mathbb{R}^{q}$ is a vector that collects all the disturbances that may affect both dynamics and measurements; $A, B, C, D$, and $E$ are real matrices of appropriate dimension. Without loss of generality, we assume that such disturbances are bounded as follows.

Assumption 1: The disturbance $t \mapsto w(t) \in \mathbb{R}^{q}$ is such that $\left|w_{i}(t)\right| \leq 1, i=1, \ldots, q$ for all $t \geq 0$.

Remark 1: The above assumption is not restrictive since, in case of scalar disturbances having modulus larger than one, we may scale the corresponding coefficients in $D$ and/or $E$ in a such a way to refer to a new scalar noise that complies with the unitary bounds.

Consider the Luenberger observer for (1) described by

$$
\dot{\hat{x}}=A \hat{x}+B u+L(y-C \hat{x})
$$

where $\hat{x}(t) \in \mathbb{R}^{n}$ is the estimate of $x(t)$ and $L \in \mathbb{R}^{n \times m}$ is the observer gain to be chosen. We need to assume the following.

Assumption 2: The pair $(A, C)$ is detectable.

The assumption above allows to construct an observer with an asymptotically stable dynamics of the estimation error $e(t):=x(t)-\hat{x}(t) \in \mathbb{R}^{n}$ in a noise-free setting but, in the presence of disturbances, the observer design is required to aim at noise rejection.

Using (1) and (2), the dynamics of the estimation error reads

$$
\dot{e}=(A-L C) e+(D-L E) w .
$$

In line with [4], the estimation error is said to be quadratically bounded with Lyapunov function $V: \mathbb{R}^{n} \rightarrow[0,+\infty)$ if

$$
V(e)>1 \Rightarrow \dot{V}(e)<0
$$

for all $w_{i} \in \mathbb{R}$ s.t. $w_{i} \in[-1,1], i=1, \ldots, q$. Using a quadratic Lyapunov function $V(e)=e^{\top} P e$ with square $n \times n$ matrix $P>0$, the above definition becomes

$$
\begin{aligned}
e^{\top} P e>1 & \Rightarrow 2 e^{\top} P((A-L C) e \\
& +(D-L E) w)<0, \forall w \in[-1,1]^{q} .
\end{aligned}
$$

Owing to (4), the set $\mathcal{E}_{P}:=\left\{e \in \mathbb{R}^{n}: e^{\top} P e \leq 1\right\}$ turns out to be positively invariant, it contains the reachable set from the origin, and it is attractive (i.e., if the error is out of $\mathcal{E}_{P}$, it approaches $\mathcal{E}_{P}$ asymptotically) [4]. Moreover, the error is upper bounded as follows:

$$
|e(t)|^{2} \leq \frac{1}{\lambda_{\min }(P)} \max \left\{e(0)^{\top} P e(0), 1\right\}
$$

for all $t \geq 0$. Clearly, such a bound combines the transient and steady-state conditions. At steady state, we have

$$
|e(t)| \leq 1 / \sqrt{\lambda_{\min }(P)}
$$

Fig. 1 pictorially depicts the various subsets of the estimation error space involved by (5) and (6).

Based on the aforesaid, we can state the following.

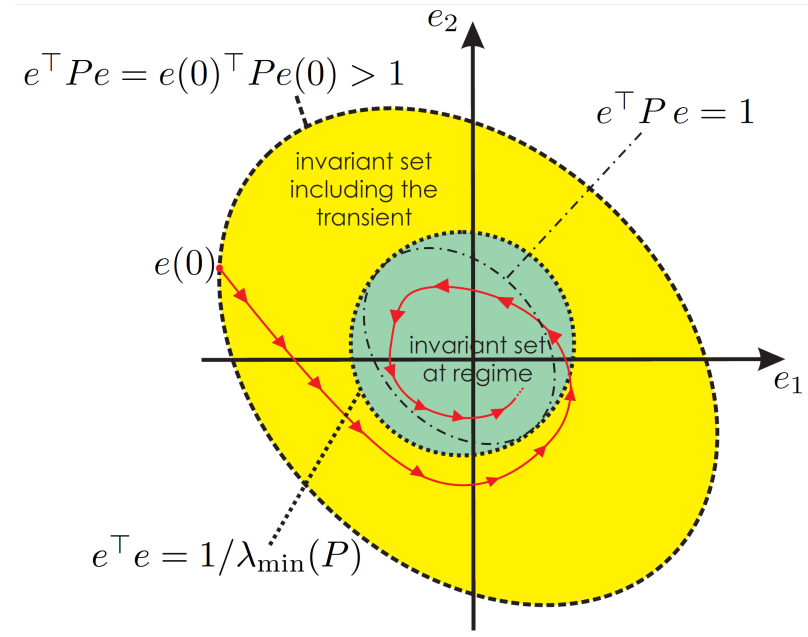

Fig. 1. The invariant set corresponding to the nominal steady-sate conditions is the set given by $e \in \mathcal{E}_{P}$, i.e., all the points inside the dotted-dashed ellipsoid. Such a set is contained inside the green ball centered in the origin since $e \in \mathcal{E}_{P}$ implies (6). i.e., $|e| \leq 1 / \sqrt{\lambda_{\min }(P)}$. The set $\mathcal{E}_{P}$ is attractive: if the initial estimation error is out of $\mathcal{E}_{P}$ (i.e., $e(0)^{\top} P e(0)>1$ ), $e(t)$ enters $\mathcal{E}_{P}$ in finite time.

Theorem 1: The estimation error is quadratically bounded if there exist $P>0, Y \in \mathbb{R}^{n \times m}, \alpha \in \mathbb{R}^{q}$ with $\alpha_{i}>0$, $i=1, \ldots, q$, and a scalar $\beta>0$ such that

$$
\left(\begin{array}{cc}
A^{\top} P-C^{\top} Y^{\top}+P A-Y C+\beta P & P D-Y E \\
\star & -\operatorname{diag}(\alpha)
\end{array}\right)<0
$$

$\sum_{i=1}^{q} \alpha_{i}-\beta \leq 0$

with $L=P^{-1} Y$.

Proof. We need to verify that the estimation error is quadratically bounded with $V(e):=e^{\top} P e$ as a Lyapunov function, i.e., (4). Using [11, S-procedure, p. 23], it follows that

$$
\begin{aligned}
& \dot{V}(e)=e^{\top}\left[(A-L C)^{\top} P+P(A-L C)\right] e \\
& +w^{\top}\left(D^{\top}-E^{\top} L^{\top}\right) P e+e^{\top} P(D-L E) w<0
\end{aligned}
$$

holds for all $e \in \mathbb{R}^{n}$ s.t. $V(e)>1$ (i.e., $-e^{\top} P e+1<0$ ) and all $w_{i} \in \mathbb{R}$ s.t. $w_{i}^{2} \leq 1$ (i.e., $w_{i}^{2}-1<0$ ), $i=1, \ldots, q$, if there exist $\alpha_{i}>0, i=1, \ldots, q$, and $\beta>0$ s.t.

$$
\begin{aligned}
& e^{\top}\left[(A-L C)^{\top} P+P(A-L C)\right] e+w^{\top}\left(D^{\top}-E^{\top} L^{\top}\right) P e \\
& +e^{\top} P(D-L E) w+e^{\top} \beta P e-\sum_{i=1}^{q} \alpha_{i} w_{i}^{2}+\sum_{i=1}^{q} \alpha_{i}-\beta \leq 0 .
\end{aligned}
$$

Such a condition is satisfied if, using $Y=P L$, we impose (7a) to account for the first terms in (8) and similarly (7b) for the last one.

Based on Theorem 1, it is convenient to keep a small steadystate error (6) by increasing $\lambda_{\min }(P)$ as much as possible. This 
TABLE I

DESIGN PROCEDURE

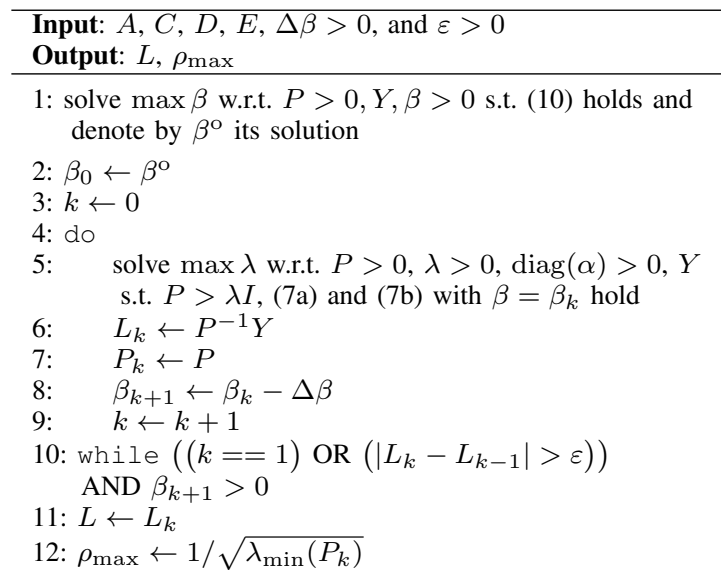

design is pursued by maximizing $\lambda$ subject to the LMI $P>\lambda I$ as follows:

$$
\begin{gathered}
\max \lambda \text { w.r.t. } P, Y, \alpha, \beta, \lambda \\
\text { s.t. } P>0, \lambda>0, \operatorname{diag}(\alpha)>0, \beta>0, \\
P>\lambda I,(7 \mathrm{a}), \text { and }(7 \mathrm{~b}) \text { hold. }
\end{gathered}
$$

This problem is not in an LMI due to the term $\beta P$ in (7a) but notice that the stability conditions reduce to the satisfaction of the inequality

$$
A^{\top} P-C^{\top} Y^{\top}+P A-Y C+\beta P<0
$$

if $w=0$. Even this condition is not an LMI form in $P$, $Y$, and $\beta$. However, one can solve a generalized eigenvalue (GEV) problem by maximizing $\beta$ to ensure a transient as fast as possible [11]. Based on the solution of such a problem, we may take the resulting maximum $\beta$ as a starting upper bound to be iteratively reduced to get the satisfaction of (7) by maximizing $\lambda_{\min }(P)$ as in (9). The design procedure is summarized in Table I, where $\varepsilon>0$ is the admitted tolerance. The gain $L$ is the final result of the procedure together with the steady-state bound $\rho_{\max }$.

\section{B. PWL systems}

The approach presented so far can be extended to a wider class of systems. Toward this end, consider the PWL systems subject to instantaneous switching described by

$$
\begin{aligned}
& \dot{x}=A_{\sigma} x+B u+D w \\
& y=C_{\sigma} x+E w \\
& \sigma\left(t^{+}\right)=F\left(x\left(t^{-}\right), u\left(t^{-}\right)\right)
\end{aligned}
$$

where $t \geq 0 ; \sigma(t) \in \Sigma:=\{1, \ldots, s\}$ represents a discrete state, which will be denoted as "mode" of the system; $(x, u) \mapsto$ $F(x, u) \in \Sigma$ is the impulsive mapping accounting for the underlying switching law, namely, it is responsible to change the system mode depending on both the state and input at the switching time. Likewise for (1), all the matrices in (11) are known as well as the impulsive mapping. The knowledge of $F(x, u)$ does not mean we know the mode of the system since only $y(t)$ is available at any time $t \geq 0$, which is just a linear combination of state variables subject to measurement noises.

Example 1: Consider the case study presented in [22] about two cascaded interconnected plants subject to mutual functional dependencies. The parameters $\mu_{1}\left(\mu_{2}\right)$ and $\lambda_{1}\left(\lambda_{2}\right)$ denote the recovery and service loss rates of the first (second) plant (see Fig. 2). The state variables $x_{1}$ and $x_{2}$ describe the quality of service of the first and second plant, respectively. In practice, if each of such variables belong to the range $[0, r]$, the service is adequately provided, while the overcoming of threshold $r$ due to the disturbances $d_{1}$ and $d_{2}$ corresponds to loss of the minimum quality of service. Thus, the change of state from functioning to non-functioning can be regarded as a mode switching. Fig. 3 illustrates the essential of such a system with four modes.

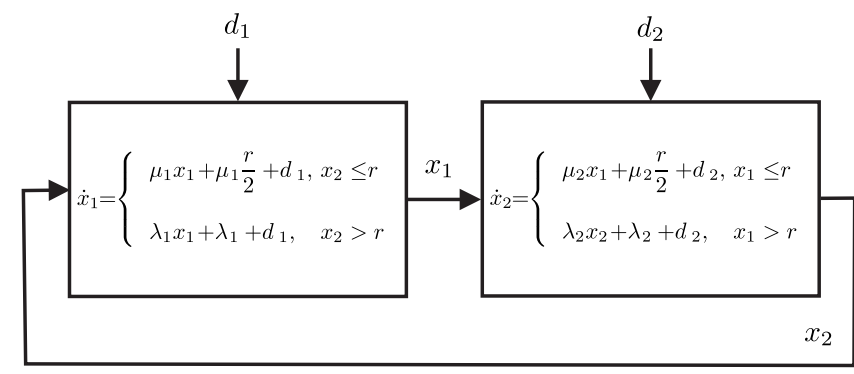

Fig. 2. Block scheme of two cascaded subsystems. The service state $x_{1}$ of the first block is input of the second block, whose state $x_{2}$ is fed back in turn.
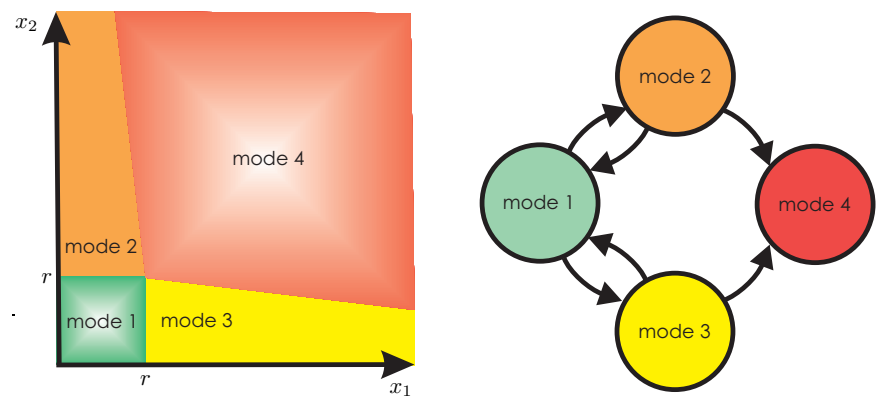

Fig. 3. Sketch of state space partitioning and finite state machine with mode transition, where $\Sigma=\{1,2,3,4\}$.

To estimate the state of (11), we consider the switching Luenberger observer given by

$$
\begin{aligned}
& \dot{\hat{x}}=A_{\hat{\sigma}} \hat{x}+B u+L_{\hat{\sigma}}\left(y-C_{\hat{\sigma}} \hat{x}\right) \\
& \hat{\sigma}\left(t^{+}\right)=F\left(\hat{x}\left(t^{-}\right), u\left(t^{-}\right)\right)
\end{aligned}
$$

where $\hat{x}(t) \in \mathbb{R}^{n}$ is the state estimate of $x(t)$. The mode is predicted according to the impulsive law (12b) and such a prediction is used to estimate the continuous state in (12a). Concerning stability, the following theorem holds.

Theorem 2: If there exist $P>0$ and $Y_{i} \in \mathbb{R}^{n \times m}$ for $i=$ $1, \ldots, s, \alpha \in \mathbb{R}^{q}$ with $\alpha_{i}>0, i=1, \ldots, q$, and a scalar $\beta>0$ 
such that

$$
\begin{aligned}
& P>I \\
& \left(\begin{array}{cc}
A_{i}^{\top} P-C_{i}^{\top} Y_{i}^{\top}+P A_{i}-Y_{i} C_{i}+\beta P & P D-Y_{i} E \\
\star & -\operatorname{diag}(\alpha)
\end{array}\right)<0 \\
& i=1, \ldots, s \\
& \sum_{i=1}^{q} \alpha_{i}-\beta \leq 0
\end{aligned}
$$

and the problem

$$
\begin{aligned}
& \min \lambda \text { s.t. } \lambda \geq 0, \text { and } \\
& \begin{array}{ccc}
P & P A_{i}-P A_{j}-Y_{j} C_{i}+Y_{j} C_{j} \\
\star & \lambda I & \\
& & i, j=1, \ldots, s, i \neq j
\end{array}
\end{aligned}
$$

admits $\lambda=0$ as solution, then the estimation error given by (12) with $L_{i}=P^{-1} Y_{i}, i=1, \ldots, s$, is quadratically bounded. Proof. The error dynamics is given by

$$
\begin{aligned}
\dot{e} & =\left(A_{\hat{\sigma}}-L_{\hat{\sigma}} C_{\hat{\sigma}}\right) e+\left(D-L_{\hat{\sigma}} E\right) w+\left(A_{\sigma}-A_{\hat{\sigma}}\right. \\
& \left.-L_{\hat{\sigma}}\left(C_{\sigma}-C_{\hat{\sigma}}\right)\right) x .
\end{aligned}
$$

Clearly, the state does not affect (15) if the matrix $A_{\sigma}-A_{\hat{\sigma}}-$ $L_{\hat{\sigma}}\left(C_{\sigma}-C_{\hat{\sigma}}\right)$ is null for any $\sigma, \hat{\sigma} \in \Sigma$ with $\sigma \neq \hat{\sigma}$. Such a decoupling ensures that the estimation error is quadratically bounded and can be obtained by a suitable choice of the gains via the minimization of $\left|A_{i}-A_{j}-L_{j}\left(C_{i}-C_{j}\right)\right|$. If

$$
\left(A_{i}-A_{j}-L_{j}\left(C_{i}-C_{j}\right)\right)\left(A_{i}-A_{j}-L_{j}\left(C_{i}-C_{j}\right)\right)^{\top} \leq \lambda I
$$

for $i, j=1, \ldots, s, i \neq j$, the minimization of $\lambda \in[0,+\infty)$ guarantees to minimize $\left|A_{i}-A_{j}-L_{j}\left(C_{i}-C_{j}\right)\right|$. Using the Schur lemma and pre-multiplying and post-multiplying for $\operatorname{diag}(P, I)$, (16) turns to be equivalent to

$$
\left(\begin{array}{cc}
P P & P A_{i}-P A_{j}-Y_{j} C_{i}+Y_{j} C_{j} \\
\star & \lambda I
\end{array}\right) \geq 0
$$

and thus to (14b) using $P P>P$ owing to (13a). Following the same reasoning of the proof of Theorem 1, it follows that QB holds for the estimation error if $\lambda=0$ solves (14).

Remark 2: If the solution of (14) is given by $\lambda=0$, we obtain the perfect decoupling of the error dynamics. If the solution is greater than zero, QB does not hold and we have only an imperfect decoupling. However, the estimation is bounded if the state trajectories are bounded and the design can be accomplished without the constraints (13a) and (14b), namely, by using only (13b) and (13c). In special cases, the problem above can be more easily treated (see, e.g, [14]). For example, the use of a high gain in the transient provides a large bandwidth, which is unsuitable at steady state, whereas instead a small gain is preferable to avoid the amplification of the measurement noises. This motivates the use of a switchinggain estimator [23], which can be studied for LTI systems in the proposed framework with the use of two gains, i.e., $L_{\mathrm{tr}}$ and $L_{\text {st }}$ for transient and steady state, respectively. The stability analysis in such a case turns out to be much simpler since the disturbing term depends on either $A_{\mathrm{tr}}-A_{\mathrm{st}}-L_{\mathrm{st}}\left(C_{\mathrm{tr}}-C_{\mathrm{st}}\right)$ or $A_{\mathrm{st}}-A_{\mathrm{tr}}-L_{\mathrm{tr}}\left(C_{\mathrm{st}}-C_{\mathrm{tr}}\right)$, which are both null matrices as $A_{\mathrm{tr}}=A_{\mathrm{st}}=A$ and $C_{\mathrm{tr}}=C_{\mathrm{st}}=C$. Notice that in general the error is not asymptotically stable to zero in a noise-free setting. This depends on the possible wrong identification of the system mode, which cannot be correctly identified at each time instant but only after some delay, even in case all the pairs $\left(A_{i}, C_{i}\right)$ are observable [24], [25].

\section{EXTENSION TO LIPSCHITZ SYSTEMS}

Instead of (1), let us consider the systems described by

$$
\begin{aligned}
& \dot{x}=A x+f(x, u)+D w \\
& y=C x+E w
\end{aligned}
$$

where $t \geq 0$ and the function $f: \mathbb{R}^{n} \times \mathbb{R}^{p} \rightarrow \mathbb{R}^{n}$ is Lipschitz. More specifically, we assume the following.

Assumption 3: There exists $k_{f}>0$ such that

$$
\left|f\left(x^{\prime}, u\right)-f\left(x^{\prime \prime}, u\right)\right| \leq k_{f}\left|x^{\prime}-x^{\prime \prime}\right|, x^{\prime}, x^{\prime \prime} \in \mathbb{R}^{n}
$$

for all $u \in \mathbb{R}^{p}$.

In addition, Assumptions 1 and 2 hold. Consider the Luenberger observer

$$
\dot{\hat{x}}=A \hat{x}+f(\hat{x}, u)+L(y-C \hat{x})
$$

where the gain $L \in \mathbb{R}^{n \times m}$ must be chosen in such a way to ensure the $\mathrm{QB}$ of the estimation error. The dynamics of the estimation error is given by

$$
\dot{e}=(A-L C) e+f(x, u)-f(\hat{x}, u)+(D-L E) w .
$$

Theorem 3: The estimation error is quadratically bounded if there exist $P>0, Y \in \mathbb{R}^{n \times m}, \alpha \in \mathbb{R}^{q}$ with $\alpha_{i}>0$, $i=1, \ldots, q$, and scalars $\beta>0, \chi>0$ such that

$$
\begin{aligned}
& \left(\begin{array}{ccc}
Q & P & P D-Y E \\
\star & -\chi I & 0 \\
\star & \star & -\operatorname{diag}(\alpha)
\end{array}\right)<0 \\
& \sum_{i=1}^{q} \alpha_{i}-\beta \leq 0
\end{aligned}
$$

where $Q:=A^{\top} P-C^{\top} Y^{\top}+P A-Y C+\beta P+\chi k_{f}^{2} I$ and $L=P^{-1} Y$.

Proof. Consider the Lyapunov function $V(e):=e^{\top} P e$. From (17) and (18), it follows that

$$
\begin{aligned}
& \dot{V}(e)=e^{\top}\left[(A-L C)^{\top} P+P(A-L C)\right] e \\
& +(f-\hat{f})^{\top} P e+e^{\top} P(f-\hat{f}) \\
& +w^{\top}\left(D^{\top}-E^{\top} L^{\top}\right) P e+e^{\top} P(D-L E) w<0
\end{aligned}
$$

where $f:=f(x, u)$ and $\hat{f}:=f(\hat{x}, u)$, for the sake of brevity. Following the same reasoning of Theorem 1, we apply [11, S-procedure, p. 23] with, in addition,

$$
(f-\hat{f})^{\top}(f-\hat{f})-k_{f}^{2}|e|^{2} \leq 0
$$

from Assumption 3 for some $\chi>0$ and obtain that QB holds by getting $\dot{V}(e)<0$ if (20a) and (20b) are satisfied. 
It is now possible to extend the previous result to the Lipschitz PWL systems described by

$$
\begin{aligned}
& \dot{x}=A_{\sigma} x+f_{\sigma}(x, u)+D w \\
& y=C_{\sigma} x+E w \\
& \sigma\left(t^{+}\right)=F\left(x\left(t^{-}\right), u\left(t^{-}\right)\right)
\end{aligned}
$$

by using the Luenberger observer given by

$$
\begin{aligned}
& \dot{\hat{x}}=A_{\hat{\sigma}} \hat{x}+f_{\hat{\sigma}}(\hat{x}, u)+L_{\hat{\sigma}}\left(y-C_{\hat{\sigma}} \hat{x}\right) \\
& \hat{\sigma}\left(t^{+}\right)=F\left(\hat{x}\left(t^{-}\right), u\left(t^{-}\right)\right)
\end{aligned}
$$

where $t \geq 0$ and the functions $f_{i}: \mathbb{R}^{n} \times \mathbb{R}^{p} \rightarrow \mathbb{R}^{n}$ satisfy the usual Lipschitz assumption, as follows.

Assumption 4: There exists $\bar{k}_{f}>0$ such that

$$
\left|f_{i}\left(x^{\prime}, u\right)-f_{i}\left(x^{\prime \prime}, u\right)\right| \leq \bar{k}_{f}\left|x^{\prime}-x^{\prime \prime}\right|, x^{\prime}, x^{\prime \prime} \in \mathbb{R}^{n}
$$

for all $u \in \mathbb{R}^{p}$ and $i=1, \ldots, s$.

Thus, we can state the following.

Theorem 4: If there exist $P>0$ and $Y_{i} \in \mathbb{R}^{n \times m}$ for $i=$ $1, \ldots, s, \alpha \in \mathbb{R}^{q}$ with $\alpha_{i}>0, i=1, \ldots, q$, and scalars $\beta>0$, $\chi>0$ such that

$$
\begin{aligned}
& P>I \\
& \left(\begin{array}{ccc}
Q_{i} & P & P D-Y E \\
\star & -\chi I & 0 \\
\star & \star & -\operatorname{diag}(\alpha)
\end{array}\right)<0, i=1, \ldots, s \\
& \sum_{i=1}^{q} \alpha_{i}-\beta \leq 0
\end{aligned}
$$

where $Q_{i}:=A_{i}^{\top} P-C_{i}^{\top} Y_{i}^{\top}+P A_{i}-Y_{i} C_{i}+\beta P+\chi \bar{k}_{f}^{2} I$ and the problem

$$
\begin{aligned}
& \min \lambda \text { s.t. } \lambda \geq 0, \text { and } \\
& \left.\begin{array}{ccc}
P & P A_{i}-P A_{j}-Y_{j} C_{i}+Y_{j} C_{j} \\
\star & \lambda I
\end{array}\right) \geq 0 \\
& \quad i, j=1, \ldots, s, i \neq j
\end{aligned}
$$

admits $\lambda=0$ as solution, then the estimation error given by (22) with $L_{i}=P^{-1} Y_{i}, i=1, \ldots, s$, is quadratically bounded. Proof. It is straightforward by combining the proofs of Theorems 2 and 3.

Remark 3: It is worth noting that the proposed approach based on QB is quite flexible and appropriate for fault diagnosis [26]. The tools that we have introduced can be used in different scenarios. For example, the switching observer (12) for PWL systems (possibly with the extension for Lipschitz nonlinearities presented in Section III) allows to detect the presence of process faults and identify them, in the case one of the modes represents nominal dynamics, while the other modes describe some faulty dynamics (a similar scenario is illustrated in [27]). Moreover, all the proposed observers can be used in a classical model-based fault detection framework [28], by comparing a residual $r(t):=y(t)-\hat{y}(t)$, computed as the difference between the measurements $y(t)$ and the estimated output $\hat{y}(t)=C \hat{x}(t)$, with threshold $\bar{r}:=|C| \bar{e}+q|E|$ based on (6), where $\bar{e}:=1 / \sqrt{\lambda_{\min }(P)}$. Using state augmentation, bias faults may be estimated as well [12]. Contrarily to approaches based on $H_{2} / H_{\infty}$ estimation for which such bounds cannot be determined since the noises are modeled as $L_{2}$ signals, the upper bound (5) on the estimation error allows to select fault-detection thresholds able by taking into account the noise bounds in such a way to reduce false alarms. The algorithm in Table I is well-suited to optimizing such thresholds by providing less conservative results.

In the next section, we will show numerical results concerning what proposed so far.

\section{Simulation Results}

Let us consider Example 1, where in addition to the original state variables denoted by $x_{1}, x_{2} \in \mathbb{R}$, we introduce two new state variables $x_{3}, x_{4} \in \mathbb{R}$ as unknown biases affecting the the dynamics of $x_{1}$ and $x_{2}$, respectively. Such a system is thus described by a PWL model with state vector $x:=\left(x_{1}, x_{2}, x_{3}, x_{4}\right)$ and

$$
\begin{aligned}
& A_{1}=\left(\begin{array}{rrrr}
-\mu_{1} & 0 & 1 & 0 \\
0 & -\mu_{2} & 0 & 1 \\
0 & 0 & 0 & 0 \\
0 & 0 & 0 & 0
\end{array}\right) A_{2}=\left(\begin{array}{rrrrr}
-\lambda_{1} & 0 & 1 & 0 \\
0 & -\mu_{2} & 0 & 1 \\
0 & 0 & 0 & 0 \\
0 & 0 & 0 & 0
\end{array}\right) \\
& A_{3}=\left(\begin{array}{rrrr}
-\mu_{1} & 0 & 1 & 0 \\
0 & -\lambda_{2} & 0 & 1 \\
0 & 0 & 0 & 0 \\
0 & 0 & 0 & 0
\end{array}\right) A_{4}=\left(\begin{array}{rrrr}
-\lambda_{1} & 0 & 1 & 0 \\
0 & -\lambda_{2} & 0 & 1 \\
0 & 0 & 0 & 0 \\
0 & 0 & 0 & 0
\end{array}\right) \\
& C_{1}=C_{2}=C_{3}=C_{4}=\left(\begin{array}{llll}
1 & 0 & 0 & 0 \\
0 & 1 & 0 & 0
\end{array}\right) .
\end{aligned}
$$

To complete the system description, we need

$$
F(x, u)= \begin{cases}1 & \text { if } x_{1} \leq r \text { and } x_{2} \leq r \\ 2 & \text { if } x_{1} \leq \min \left\{r, 1-(1-r)\left(x_{2} / r\right)^{\lambda_{1} / \mu_{2}}\right\} \\ & \text { and } x_{2}>r \\ 3 & \text { if } x_{2} \leq \min \left\{r, 1-(1-r)\left(x_{1} / r\right)^{\lambda_{2} / \mu_{1}}\right\} \\ & \text { and } x_{1}>r \\ 4 & \text { otherwise }\end{cases}
$$

for the switching dynamics [22] and

$$
D=\left(\begin{array}{rrrr}
0 & 0 & 0.1 & 0 \\
0 & 0 & 0 & 0.1 \\
0 & 0 & 0 & 0 \\
0 & 0 & 0 & 0
\end{array}\right) \quad E=\left(\begin{array}{rrrr}
0.01 & 0 & 0 & 0 \\
0 & 0.01 & 0 & 0
\end{array}\right) \text {. }
$$

The state variables $x_{3}$ and $x_{4}$ account for the regime values associated with each mode, i.e., $x_{3}=\mu_{1} r / 2$ and $x_{4}=\mu_{2} r / 2$ for mode $1, x_{3}=\lambda_{1}$ and $x_{4}=\mu_{2} r / 2$ for mode $2, x_{3}=$ $\mu_{1} r / 2$ and $x_{4}=\lambda_{2}$ for mode $3, x_{3}=\lambda_{1}$ and $x_{4}=\lambda_{2}$ for mode 4 . Note also that at regime in the absence of noises the operating point in mode 1 is just given by $x_{1}=x_{2}=$ $r / 2$, while in mode 4 such state variables tend to 1 . Thus, the estimates of the state variables may be used to detect the occurrence of a service downgrade.

We have chosen $\mu_{1}=\mu_{2}=0.2, \lambda_{1}=\lambda_{2}=0.5$, and $r=$ 0.4. Based on Theorem 2, we have solved (14) by obtaining $\lambda>0$ as solution. Since perfect decoupling is not satisfied but the state trajectories are bounded, we have addressed the 
design according to Remark 2 by using the procedure in Table I based only on (13b) and (13c) and getting

$$
\begin{aligned}
& P=\left(\begin{array}{rrrr}
80.6811 & 0 & -18.1225 & 0 \\
0 & 94.1508 & 0 & -18.1225 \\
-18.1225 & 0 & 41.3424 & 0 \\
0 & -18.1225 & 0 & 41.3424
\end{array}\right) \\
& L_{1}=\left(\begin{array}{rr}
4.4563 & 0 \\
0 & 4.4563 \\
1.8203 & 0 \\
0 & 1.8203
\end{array}\right) L_{2}=\left(\begin{array}{rr}
3.4456 & 0 \\
0 & 4.4563 \\
1.6928 & 0 \\
0 & 1.82036
\end{array}\right) \\
& L_{3}=\left(\begin{array}{rr}
4.4563 & 0 \\
0 & 3.4456 \\
1.8203 & 0 \\
0 & 1.6928
\end{array}\right) \quad L_{4}=\left(\begin{array}{rr}
3.4456 & 0 \\
0 & 3.4456 \\
1.6928 & 0 \\
0 & 1.6928
\end{array}\right) \\
& \alpha=\left(\begin{array}{l}
0.0359 \\
0.0359 \\
0.1881 \\
0.1881
\end{array}\right) \beta=0.4480 \text {. }
\end{aligned}
$$

In the following we will refer to such an estimator as QB observer, for short.

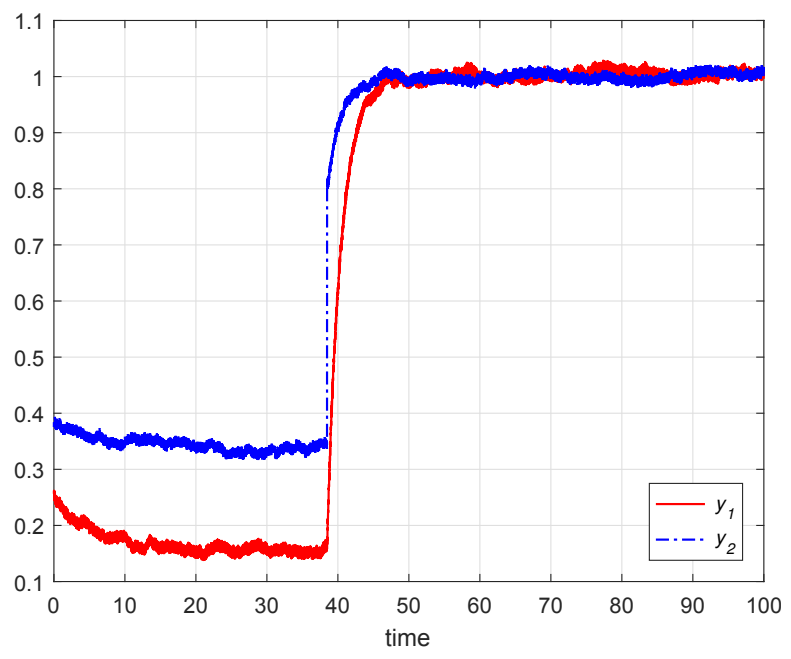

Fig. 4. Time behavior of $y_{1}$ and $y_{2}$.

Each simulation run has been initialized according to a Gaussian distribution with covariance $P_{0}=\operatorname{diag}(0.01,0.01,0.001,0.001)$ and mean equal to $\bar{x}(0)=\left(r / 2, r / 2, \mu_{1} r / 2, \mu_{2} r / 2\right)$. The noises have been generated according to an uniform distribution in the range $[-1,1]$. For the purpose of comparison with the proposed approach we have designed a switching Kalman filter (SKF, see [29], [30]) with the same mode estimator of the QB observer, i.e., (24) with the corresponding state estimates as input. The initial estimated states of the QB observer and the SKF in all the simulation runs have been taken equal to $\bar{x}(0)$. Concerning only the SKF, we have chosen the covariance matrices of the initial-state and system noise equal to $P_{0}$ and $Q=\operatorname{diag}(0.0001,0.0001,0.0001,0.0001)$, respectively. Instead, different matrices $R$ (i.e., the covariance matrix of the measurement noise) have been chosen in order to improve the SKF performances.

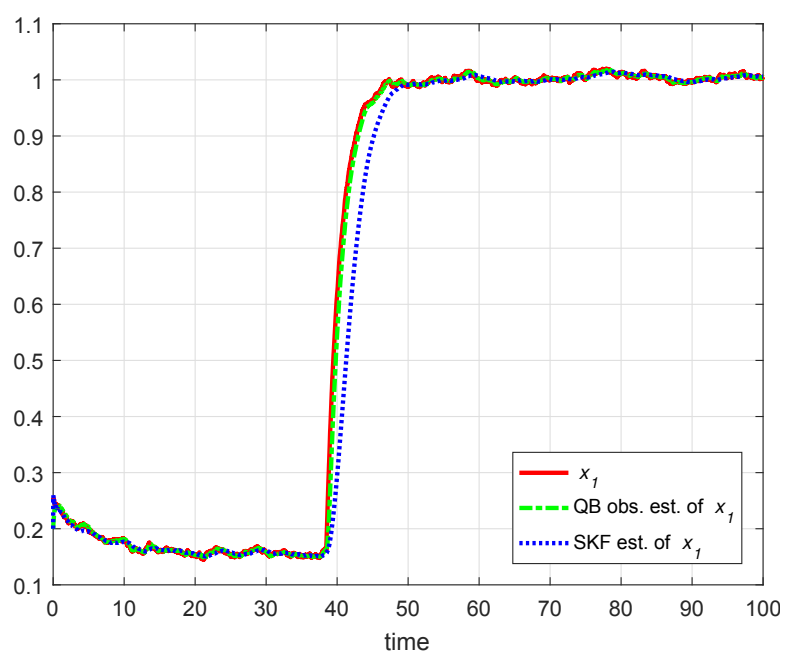

Fig. 5. Time behavior of $x_{1}$ and its estimates.

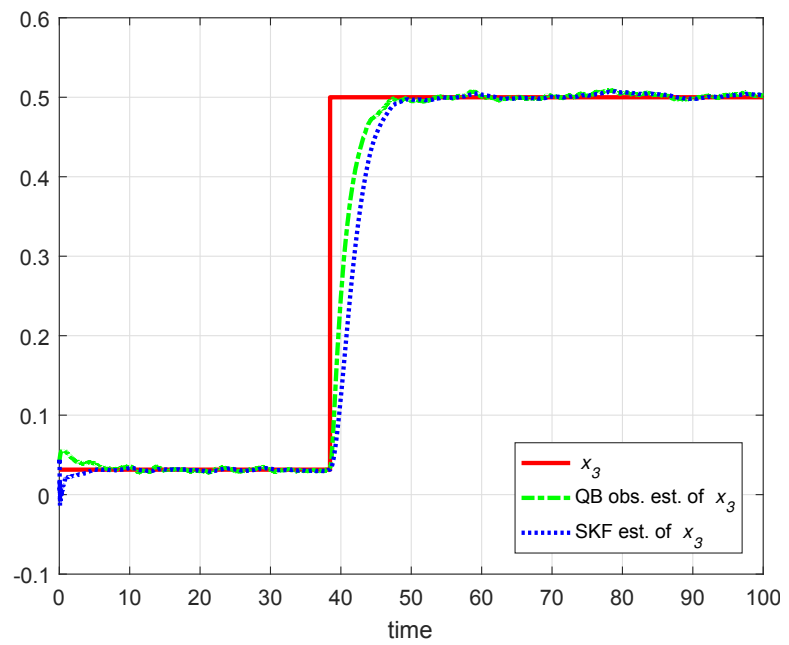

Fig. 6. Time behavior of $x_{3}$ and its estimates.

Figs. 4-6 show the result of a simulation run, in which the QB observer provides a quicker reaction to the occurrence of the fault, as compared to the SKF (only $x_{1}, x_{3}$ and their estimates are plotted since the behaviors of $x_{2}, x_{4}$ and corresponding estimates are similar). Generally speaking, the tuning of the SKF is not easy at all. Depending on the selection of covariance matrix of the measurement noises, on one hand the response to the fault may be rapid but with an estimation error very sensitive to the noises or, on the other hand, slow but more robust to noises.

As an alternative to previous mathematical description of Example 1, one can refer to an autonomous Lipschitz PWL model with two additional state variables that account for bias 
on the original dynamics, i.e.,

$$
\begin{aligned}
& A_{1}=A_{2}=A_{3}=A_{4}=\left(\begin{array}{rrrr}
0 & 0 & 1 & 0 \\
0 & 0 & 0 & 1 \\
0 & 0 & 0 & 0 \\
0 & 0 & 0 & 0
\end{array}\right) \\
& f_{1}(x)=\left(-\mu_{1} x_{1}+\mu_{1} r / 2,-\mu_{2} x_{2}+\mu_{2} r / 2,0,0\right) \\
& f_{2}(x)=\left(-\lambda_{1} x_{1}+\lambda_{1},-\mu_{2} x_{2}+\mu_{2} r / 2,0,0\right) \\
& f_{3}(x)=\left(-\mu_{1} x_{1}+\mu_{1} r / 2,-\lambda_{2} x_{2}+\lambda_{2}, 0,0\right) \\
& f_{4}(x)=\left(-\lambda_{1} x_{1}+\lambda_{1},-\lambda_{2} x_{2}+\lambda_{2}, 0,0\right) \\
& C_{1}=C_{2}=C_{3}=C_{4}=\left(\begin{array}{rrrr}
1 & 0 & 0 & 0 \\
0 & 1 & 0 & 0
\end{array}\right) \\
& D=\left(\begin{array}{llll}
0 & 0 & 0.1 & 0 \\
0 & 0 & 0 & 0.1 \\
0 & 0 & 0 & 0 \\
0 & 0 & 0 & 0
\end{array}\right) E=\left(\begin{array}{rrrr}
0.01 & 0 & 0 \\
0 & 0.01 & 0 & 0
\end{array}\right)
\end{aligned}
$$

and the same switching mapping (24). The observer (22) has been designed likewise the previous estimator but of course according to the stability conditions of Theorem 4 without perfect decoupling, i.e., using only (23b) and (23c) in the design procedure of Table I. We have obtained

$$
\begin{gathered}
P=\left(\begin{array}{rrrr}
163.0767 & 0 & -18.9876 & 0 \\
0 & 163.0767 & 0 & -18.9876 \\
0 & 7.9556 & 0 \\
-18.9876 & -18.9876 & 0 & 7.9556
\end{array}\right) \\
L_{1}=L_{2}=L_{3}=L_{4}=\left(\begin{array}{rrr}
11.7927 & 0 \\
0 & 11.7927 \\
28.1459 & 0 \\
0 & 28.1459
\end{array}\right) \\
\alpha=\left(\begin{array}{c}
0.1389 \\
0.1389 \\
0.5051 \\
0.5051
\end{array}\right) \beta=1.2880
\end{gathered}
$$

with $\bar{k}_{f}=\max \left(\mu_{1}, \mu_{2}, \lambda_{1}, \lambda_{2}\right)$. We will refer to this estimator as Lipschitz QB or LQB observer for short. The result of a simulation run is shown in Fig. 7-9.

Fig. 9 shows how good the resulting QB threshold $\rho_{\max }$ is as compared to the standard noise level and upon the occurrence of the fault, which causes a mode transition. Thus, in principle one can rely also on a decision rule based on this threshold to detect a fault.

Table II shows that the proposed approaches perform quite well as compared to the SKF (see also Fig. 10). The gain of the LQB observer turns out to be higher than that of the QB observer, which is not surprising since the Lipschitz nonlinearity demands an increase of the gain to some extent in such a way to dominate the nonlinear component of the error dynamics. The performances of the SKF are very sensitive to the choice of the matrix $R$. A "smaller" $R$ ensures a lower estimation error but at a prize of a slower reaction to the occurrence of mode switching, which entails a worse correctdetection rate. Moreover, the computational effort required by QB/LQB estimators is about $80 \%$ lower than that of the SKF since all the gain matrices of such estimators are computed

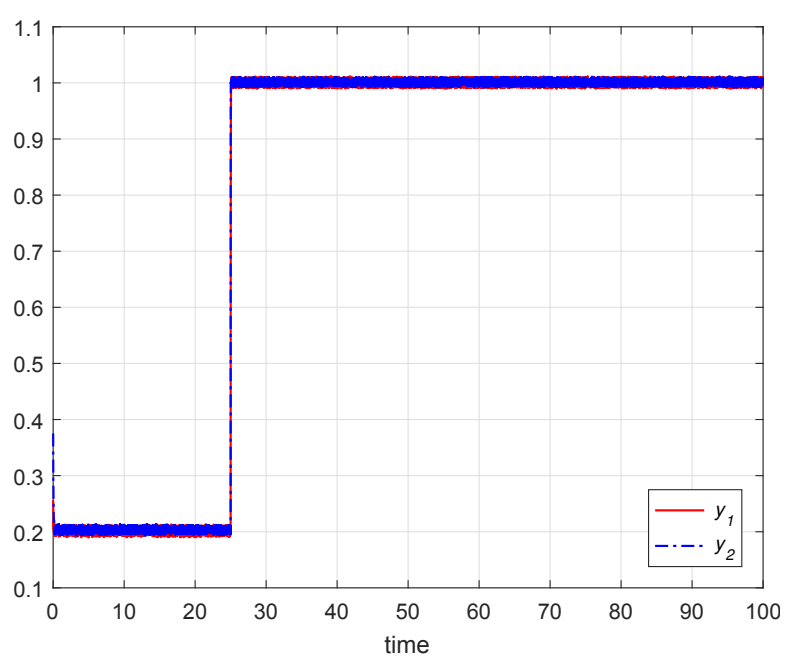

Fig. 7. Time behavior of $y_{1}$ and $y_{2}$.

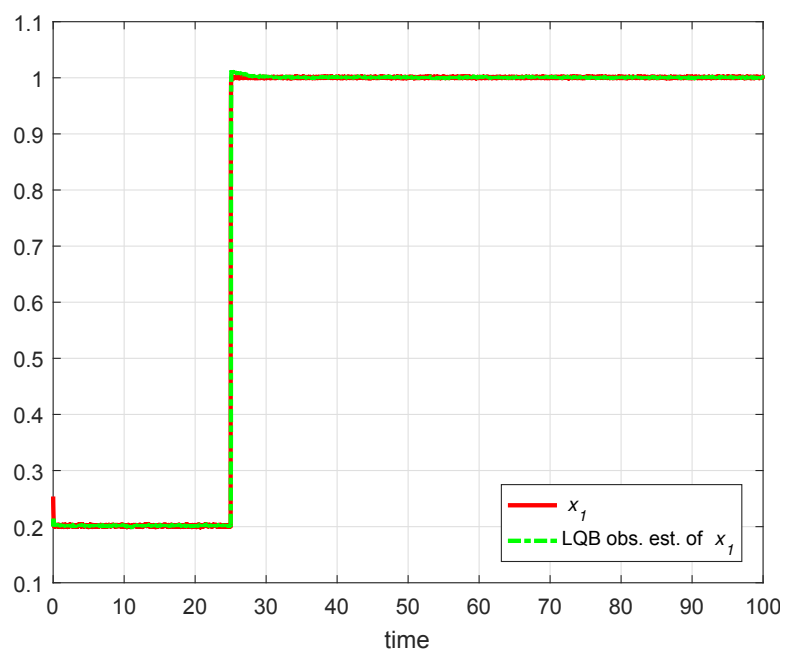

Fig. 8. Time behavior of $x_{1}$ and its estimate.

off line, while the on-line computation of the gain is required for the SKF.

TABLE II

PERCENTAGE OF CORRECT MODE ESTIMATION (PCME) AND MEDIAN OF ROOT MEAN SQUARE ERROR (RMSE) OVER 1000 SIMULATION RUNS.

\begin{tabular}{ccc} 
& PCME & RMSE median \\
\hline QB observer & 99.8 & 0.028 \\
\hline LQB observer & 99.9 & 0.048 \\
\hline SKF with $R=\operatorname{diag}(0.01,0.01)$ & 99.3 & 0.034 \\
\hline SKF with $R=\operatorname{diag}(0.1,0.1)$ & 99.6 & 0.060 \\
\hline SKF with $R=\operatorname{diag}(1,1)$ & 97.4 & 0.112 \\
\hline
\end{tabular}

\section{Conclusions}

In this paper, the problem of observer design for dynamic systems affected by bounded noises has been addressed by using the notion of quadratic boundedness for both LTI and PWL systems, even with a possible Lipschitz nonlinearity in the dynamics. The design of the estimators can be accomplished by minimizing a steady-state upper bound on the estimation error. The proposed observers perform quite well in terms 


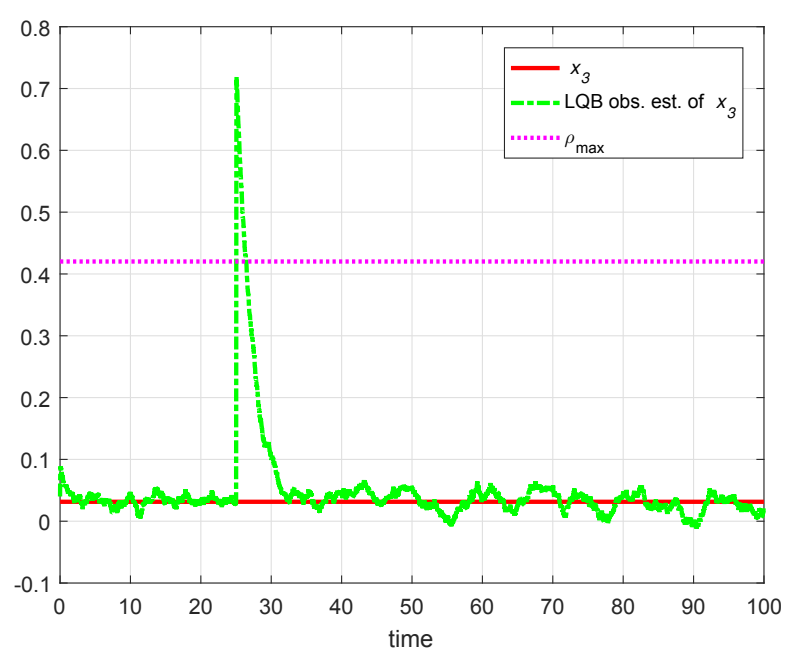

Fig. 9. Time behavior of $x_{3}$ and its estimate.

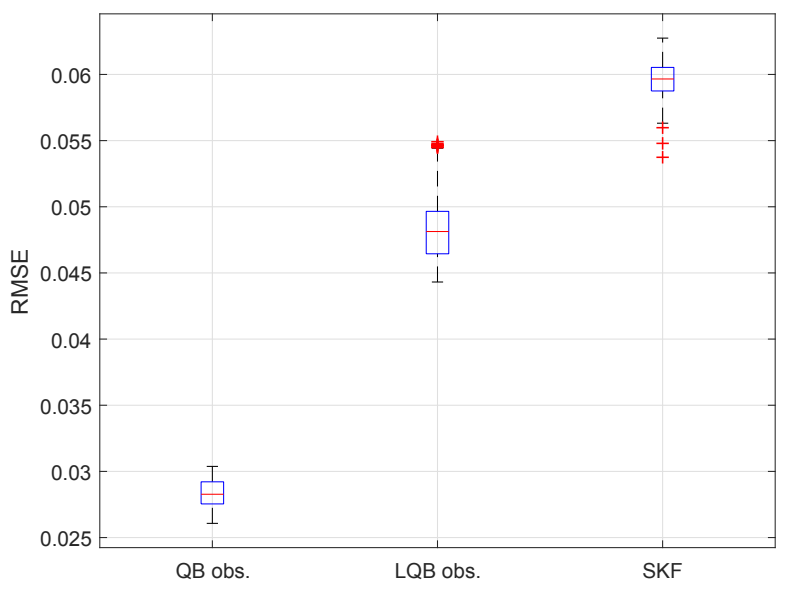

Fig. 10. Boxplots the RMSEs over 1000 simulation runs (SKF with $R=$ $\operatorname{diag}(0.1,0.1))$.

of accuracy as compared to the Kalman filter and demand a much lower computational effort. As a future work, we aim at applying the proposed approach to fault diagnosis in general and, in particular, to attack detection for cyber-physical systems [31], where we may take advantage of the possibility to account for bounds on the noises.

\section{ACKNOWLEDGMENTS}

The authors would like to thank Thomas Parisini for the fruitful discussions and suggestions that helped in the outcome of this work.

\section{REFERENCES}

[1] R. Kalman, "A new approach to linear filtering and prediction problems," Transactions of the ASME-Journal of Basic Engineering, vol. 82, no. 1, pp. 35-45, 1960.

[2] P. Khargonekar, M. Rotea, and E. Baeyens, "Mixed $H_{2} / H_{\infty}$ filtering," International Journal of Robust and Nonlinear Control, vol. 6, no. 4, pp. 313-330, 1996.

[3] D. Luenberger, "Observing the state of a linear system," IEEE Trans. on Military Electronics, vol. 8, no. 2, pp. 74-80, 1964.

[4] M. Brockman and M. Corless, "Quadratic boundedness of nominally linear systems," Int. Journal of Control, vol. 71, no. 6, pp. 1105-1117, 1998.
[5] F. C. Schweppe, Uncertain Dynamic Systems. Prentice-Hall, Englewood Cliffs, New Jersey, 1973.

[6] B. Chen and G. Hu, "Nonlinear state estimation under bounded noises," Automatica, vol. 98, pp. 159 - 168, 2018.

[7] F. Lauer, "Global optimization for low-dimensional switching linear regression and bounded-error estimation," Automatica, vol. 89, pp. $73-$ 82, 2018.

[8] B. Ding, "Quadratic boundedness via dynamic output feedback for constrained nonlinear systems in Takagi-Sugeno's form," Automatica, vol. 45, no. 9, pp. 2093 - 2098, 2009.

[9] A. Alessandri, M. Baglietto, and G. Battistelli, "On estimation error bounds for receding-horizon filters using quadratic boundedness," IEEE Trans. on Automatic Control, vol. 49, no. 8, pp. 1350-1355, 2004.

[10] — "Design of state estimators for uncertain linear systems using quadratic boundedness," Automatica, vol. 42, no. 3, pp. 497-502, 2006.

[11] S. Boyd, L. El Ghaoui, E. Feron, and V. Balakrishnan, Linear Matrix Inequalities in System and Control Theory, ser. Studies in Applied Mathematics. Philadelphia, PA: SIAM, 1994, vol. 15.

[12] A. Alessandri, F. Boem, and T. Parisini, "Model-based fault detection and estimation for linear time invariant and piecewise affine systems by using quadratic boundedness," in 57th IEEE Conference on Decision and Control, Miami, USA, 2018, pp. 5562-5567.

[13] G. Feng, "Nonsynchronized state estimation of discrete time piecewise linear systems," IEEE Trans. on Signal Processing, vol. 54, no. 1, pp. 295-303, 2006.

[14] A. Lj. Juloski, W. P. M. H. Heemels, and S. Weiland, "Observer design for a class of piecewise linear systems," International Journal of Robust and Nonlinear Control, vol. 17, no. 15, pp. 1387-1404, 2007.

[15] S. Ibrir, "Circle-criterion approach to discrete-time nonlinear observer design," Automatica, vol. 43, no. 8, pp. 1432 - 1441, 2007.

[16] A. Zemouche and M. Boutayeb, "On LMI conditions to design observers for Lipschitz nonlinear systems," Automatica, vol. 49, no. 2, pp. 585591,2013

[17] A. Alessandri and A. Rossi, "Increasing-gain observers for nonlinear systems: stability and design," Automatica, vol. 57, no. 7, pp. 180-188, 2015.

[18] A. Zemouche, R. Rajamani, G. Phanomchoeng, B. Boulkroune, H. Rafaralahy, and M. Zasadzinski, "Circle criterion-based $H_{\infty}$ observer design for Lipschitz and monotonic nonlinear systems - Enhanced LMI conditions and constructive discussions," Automatica, vol. 85, pp. 412425, 2017.

[19] A. Voelker, K. Kouramas, and E. Pistikopoulos, "Moving horizon estimation: Error dynamics and bounding error sets for robust control," Automatica, vol. 49, no. 4, pp. 943-948, 2013.

[20] M. Muller, "Nonlinear moving horizon estimation in the presence of bounded disturbances," Automatica, vol. 79, pp. 306 - 314, 2017.

[21] A. Alessandri and M. Gaggero, "Fast moving horizon state estimation for discrete-time systems using single and multi iteration descent methods," IEEE Trans. on Automatic Control, vol. 62, no. 9, pp. 4499-4511, 2017.

[22] A. Alessandri and R. Filippini, "Evaluation of resilience of interconnected systems based on stability analysis," in CRITIS 2012, Lecture Notes in Computer Sciences, B. Hämmerli, N. K. Svendsen, and J. Lopez, Eds., vol. 7722. Springer-Verlag, 2013, pp. 180-190.

[23] D. Mayne, R. Grainger, and G. Goodwin, "Nonlinear filters for linear signal models," IEE Proceedings - Control Theory and Applications, vol. 144, pp. 281-286, 1997.

[24] A. Alessandri, M. Baglietto, and G. Battistelli, "Receding-horizon estimation for switching discrete-time linear systems," IEEE Trans. on Automatic Control, vol. 50, no. 11, pp. 1736-1748, 2005.

[25] - "Luenberger observers for switching discrete-time linear systems," Int. Journal of Control, vol. 80, no. 12, p. 1931-1943, 2007.

[26] L. Li, S. Ding, J. Qiu, K. Peng, and Y. Yang, "An optimal fault detection approach for piecewise affine systems via diagnostic observers,' Automatica, vol. 85, pp. 256-263, 2017.

[27] D. M. Raimondo, F. Boem, A. Gallo, and T. Parisini, "A decentralized fault-tolerant control scheme based on active fault diagnosis," in IEEE 55th Conference on Decision and Control. IEEE, 2016, pp. 2164-2169.

[28] M. Blanke, M. Kinnaert, J. Lunze, M. Staroswiecki, and J. Schröder, Diagnosis and fault-tolerant control. Springer, 2006, vol. 2.

[29] G. Böker and J. Lunze, "Stability and performance of switching Kalman filters," Int. Journal of Control, vol. 75, pp. 1269-1281, 2002.

[30] A. Alessandri, M. Baglietto, and G. Battistelli, "A maximum-likelihood Kalman filter for switching discrete-time linear systems," Automatica, vol. 46, no. 11, pp. 1870-1876, 2010.

[31] Y. Nakahira and Y. Mo, "Attack-resilient $\mathcal{H}_{2}, \mathcal{H}_{\infty}$, and $\ell_{1}$ state estimator," IEEE Trans. on Automatic Control, vol. 63, no. 12, pp. 43534360, 2018. 